\title{
$D_{s}$ physics from fine lattices
}

$\overline{\mathrm{A}_{\text {Collaboration }}}$

DESY 08-117

$S F B / C P P-08-76$

$M S-T P-08-24$

Georg von Hippel*, Rainer Sommer

Deutsches Elektronen-Synchrotron DESY, Platanenallee 6, 15738 Zeuthen, Germany

Email: georg.von.hippeledesy.de

\section{Jochen Heitger}

Westfälische Wilhelms-Universität Münster, Institut für Theoretische Physik,

Wilhelm-Klemm-Str. 9, 48149 Münster, Germany

\section{Stefan Schaefer}

Humboldt-Universität zu Berlin, Institut für Physik, Newtonstr. 15, 12489 Berlin, Germany

\section{Nazario Tantalo}

INFN sezione di Roma “Tor Vergata”, I-00133 Roma, Italy

We present a preliminary analysis of the charm quark mass and the mass and decay constant $f_{D_{s}}$ of the $D_{s}$ meson obtained from dynamical simulations of $N_{f}=2$ Wilson QCD on the large and fine lattices simulated by the CLS effort.

Based on CLS configurations

The XXVI International Symposium on Lattice Field Theory

July 14 - 19, 2008

Williamsburg, Virginia, USA

${ }^{*}$ Speaker. 


\section{Introduction}

Leptonic decays of charmed mesons were not expected to be a channel where new physics might be found. However, new, precise experimental results by CLEO [1] show unexpectedly high rates in the decays $D_{\mathrm{s}} \rightarrow \tau \nu, \mu \nu$ compared to estimates from decay constants in the quenched approximation. The HPQCD collaboration found the effect of (rooted) dynamical staggered quarks to be significantly smaller than the difference between experiment and the quenched calculations [2] ${ }^{1}$. Is this evidence for new physics [3], is it a statistical fluctuation or underestimate of systematics in the experiment, or is it a systematic effect unaccounted for by the errors quoted in [细? We are aiming at a precise calculation of $f_{D_{s}}$ as well as other observables such as the charm quark mass, using the $N_{f}=2$ CLS configurations which reach small lattice spacings, where the charm quark mass in lattice units is really small. Here we describe first, encouraging, steps. In particular, we find small lattice spacing effects for $\mathrm{O}(a)$ improved Wilson quarks.

\section{The CLS coordinated lattice simulations effort}

Coordinated Lattice Simulations (CLS) is a community effort to bring together the human and computer resources of several teams in Europe interested in lattice QCD. CLS member teams are located at CERN, in Germany (Berlin, DESY/Zeuthen, Mainz), Italy (Rome) and Spain (Madrid, Valencia). All CLS simulations use M. Lüscher's implementation of the DD-HMC algorithm [円] to efficiently simulate $N_{f}=2$ Wilson QCD with non-perturbative $\mathrm{O}(a)$ improvement on a variety of computer architectures ranging from PC clusters to the BlueGene/P at NIC/Forschungszentrum Jülich.

Table 1 shows the existing CLS ensembles. For this initial study of heavy quark physics on these ensembles we will use the D2, E6 and Q4 ensembles in order to get an idea of the size of the sea quark mass and lattice spacing effects.

\section{Setting the scale}

A final determination of the lattice scale, e.g. via $m_{\Omega}$, is not yet available for the CLS ensembles. In [5] Del Debbio et al. determined the scale on the coarsest $(\beta=5.3)$ CLS ensembles to be $a=0.0784(10) \mathrm{fm}$ via a combination of $m_{K}$ and $m_{K^{*}}$. We use this as our value for $a$ on the D2 and E6 ensembles, and run it to $\beta=5.7$ for the Q4 ensemble by means of the scale $L^{*}$ defined in [6] via $\bar{g}^{2}\left(L^{*}\right)=5.5$ in the Schrödinger functional scheme. Specifically, we use the linear fit $\log \left(L^{*} / a\right)=2.3338+1.4025(\beta-5.5) \pm 0.02$.

Since the uncertainty about the scale is an important source of error at $\beta>5.3$, and the somewhat unphysical determination of the scale may be considered as a source of an unquantifiable systematic error even at $\beta=5.3$, a more accurate determination of the scale is certainly a priority in order to make accurate predictions.

\footnotetext{
${ }^{1}$ Note that a relatively new discretization for the charm quarks is used.
} 


\section{Measurements and Analysis}

We use 6 time-localized $U(1)$ noise sources per configuration to measure the correlators $C_{A A}$, $C_{A P}, C_{P A}$ and $C_{P P}$, where

$$
C_{X Y}\left(x_{0}\right)=-a^{3} \sum_{\mathbf{x}}\left\langle X_{12}(x) Y_{21}(0)\right\rangle
$$

$P_{i j}=\bar{q}_{i} \gamma_{5} q_{j}$ and $A_{i j}=\bar{q}_{i} \gamma_{0} \gamma_{5} q_{j}$, on 61 configurations of D2, 28 configurations of E6, and 31 configurations of Q4, performing a fully correlated error analysis using the Jackknife method in each case.

As in [7], we define the effective mass $M_{\text {eff }}\left(x_{0}\right)$ via

$$
\frac{g\left(M_{\mathrm{eff}}\left(x_{0}\right), x_{0}-a\right)}{g\left(M_{\mathrm{eff}}\left(x_{0}\right), x_{0}\right)}=\frac{C\left(x_{0}-a\right)}{C\left(x_{0}\right)}
$$

where $g(M, x)=e^{-M x}+e^{-M(T-x)}$. Effective matrix elements are defined as e.g.

$$
G_{P S, \mathrm{eff}}\left(x_{0}\right)=\sqrt{\frac{C_{P P}\left(x_{0}\right) M_{\mathrm{eff}}\left(x_{0}\right)}{g\left(M_{\mathrm{eff}}\left(x_{0}\right), x_{0}\right)}} .
$$

We also define the PCAC quark mass as

$$
\left(m_{s}+m_{c}\right)=m\left(x_{0}\right)=\frac{\frac{1}{2}\left(\partial_{0}+\partial_{0}^{*}\right) C_{P A}\left(x_{0}\right)+c_{A} a \partial_{0} \partial_{0}^{*} C_{P P}\left(x_{0}\right)}{C_{P P}\left(x_{0}\right)},
$$

which needs to be renormalized and $\mathrm{O}(a)$ improved as $m(\mu)=Z_{A} Z_{P}^{-1}(\mu)\left(1+\frac{1}{2}\left(b_{A}-b_{P}\right)\left(m_{q} a\right)\right) m$. In terms of these quantities, the (renormalized) pseudoscalar decay constant is defined as $F_{P S}=$ $Z_{A}\left(1+\frac{1}{2} b_{A}\left(m_{q} a\right)\right) \frac{m G_{P S}}{M_{P S}^{2}}$.

We use non-perturbative renormalization wherever possible, in particular for $c_{A}$ [8], $Z_{A}$ [9], $Z_{P}$ [10], and $b_{A}-b_{P}$ [11]. Perturbative (one-loop) renormalization is used only for $b_{A}$, where no non-perturbative results are available. We translate the PCAC masses into the RGI masses through non-perturbative running in the Schrödinger functional scheme as in [10].

\begin{tabular}{lllll}
\hline Id & Size & $a[\mathrm{fm}]$ & $\kappa$ & $\mathrm{MD} \tau$ \\
\hline D1 & $48 \times 24^{3}$ & 0.08 & 0.13550 & 2575 \\
D2 & & & 0.13590 & 2565 \\
D3 & & & 0.13610 & 2520 \\
D4 & & & 0.13620 & 2505 \\
D5 & & & 0.13625 & 2510 \\
\hline E1 & $64 \times 32^{3}$ & 0.08 & 0.13550 & 2672 \\
E2 & & & 0.13590 & 2512 \\
E3 & & & 0.13605 & 2512 \\
E4 & & & 0.13610 & 2497 \\
E5 & & & 0.13625 & 2656 \\
E6 & & & 0.13635 & 4960 \\
\hline
\end{tabular}

\begin{tabular}{lllll}
\hline Id & Size & $a[\mathrm{fm}]$ & $\kappa$ & MD $\tau$ \\
\hline M1 & $64 \times 32^{3}$ & 0.06 & 0.13620 & 4055 \\
M2 & & & 0.13630 & 3772 \\
M3 & & & 0.13640 & 2980 \\
M4 & & & 0.13650 & 3790 \\
M5 & & & 0.13660 & 2570 \\
\hline P1 & $96 \times 48^{3}$ & 0.04 & 0.13620 & 1702 \\
P2 & & & 0.13630 & started \\
P3 & & & 0.13640 & started \\
\hline Q4 & $128 \times 64^{3}$ & 0.04 & 0.13640 & $1450+$ \\
Q5 & & & 0.13650 & $1120+$ \\
Q6 & & & 0.136575 & started \\
\hline
\end{tabular}

Table 1: The existing and running CLS ensembles; ensembles used in this study are shown in boldface. The molecular dynamics time $\tau$ is given in MD units after thermalisation; trajectory length is typically $\tau=0.5$. 


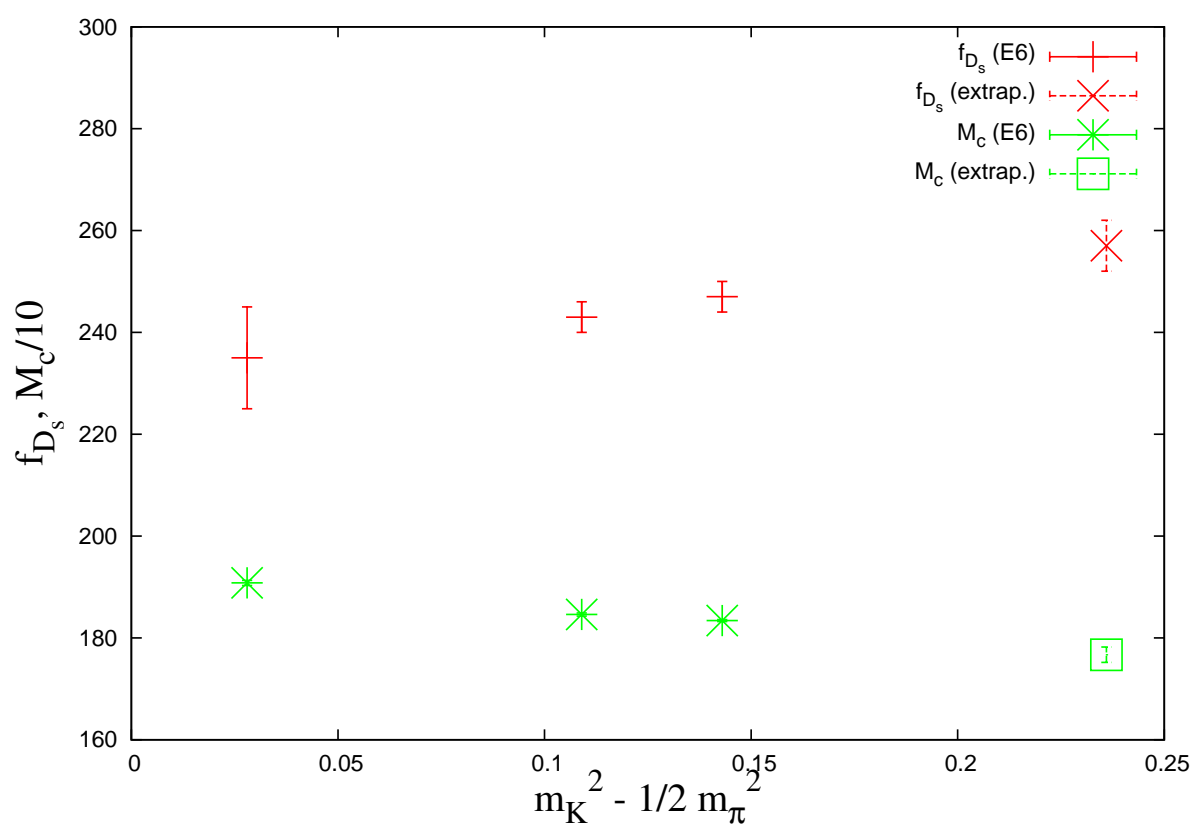

Figure 1: The quark mass $M_{c}$ (divided by 10 for scale) and $f_{D_{s}}$ as a function of $\left(m_{K}^{2}-\frac{1}{2} m_{\pi}^{2}\right)$ on the E6 ensemble, together with the linearly extrapolated values at the physical point.

\section{Preliminary results}

\section{1 $D_{s}$ masses and $M_{c}$}

Using two heavy quark masses on each ensemble, and two light/strange-quark masses on E6 and Q4, three light/strange quark masses on D2, and one additional light quark mass on E6, we measure the masses of all possible mass combinations of pseudoscalar mesons, as well as the corresponding PCAC quark masses. From the latter, we extract the RGI mass $M_{c}$ as described in [10, 12].

To extract a physical value for $M_{c}$ from these data, we first interpolate $M_{c}$ linearly as a function of $M_{D_{s}}$ to get $M_{c}$ as a function of the light and strange quark masses. This we treat as a function of $\left(m_{K}^{2}-\frac{1}{2} m_{\pi}^{2}\right)$, a $\chi$ PT-inspired proxy of the strange quark mass, and extrapolate to the physical point $\left(m_{K}^{2}-\frac{1}{2} m_{\pi}^{2}\right)=0.236(\mathrm{GeV})^{2}$ as shown in fig. 1.

Our results are $M_{c}=1694(3)(34) \mathrm{MeV}, 1767(15)(35) \mathrm{MeV}$ and 1666(1)(33) MeV on the D2, E6 and Q4 ensembles, respectively. We note that the lattice spacing dependence (the $1 \%$ difference between the D2 and Q4 ensembles) is small, but the sea quark mass dependence (the $4 \%$ difference between the E6 and D2 ensembles) is noticeable.

\subsection{Lattice spacing effects in $f_{D_{s}}$}

To get an estimate of what the lattice spacing effects on the decay constant of the $D_{s}$ are likely to be, we define an (unphysical) reference point at $m_{\pi}^{\text {ref }}=m_{K}^{\text {ref }}=618 \mathrm{MeV}, m_{D}=1968 \mathrm{MeV}$, to compare results obtained at different lattice spacings. This point is realized directly on the D2 ensemble, where $L^{*} f_{K}^{\mathrm{ref}}=0.541(16)(11)$, and $L^{*} f_{D}^{\mathrm{ref}}=0.805(12)(16)$. On the Q4 ensemble, we 


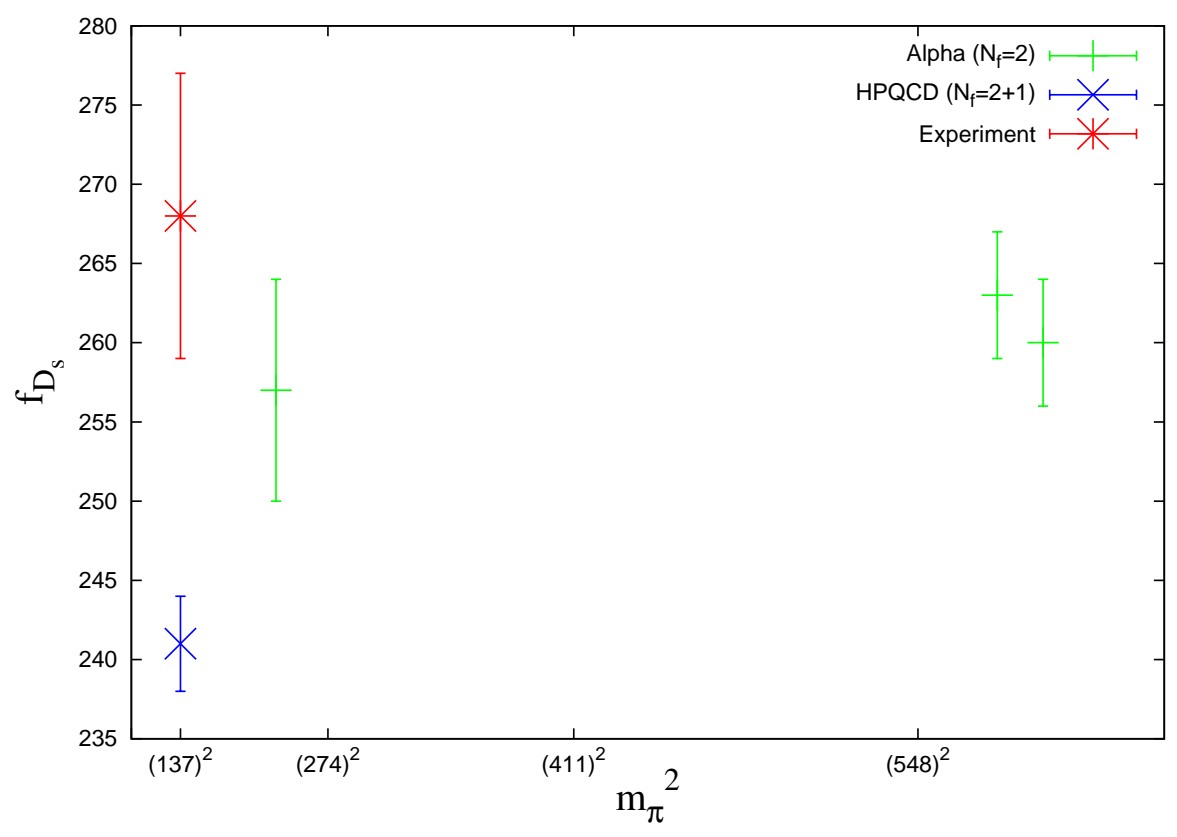

Figure 2: Summary plot showing the dependence of our results on the pion (sea quark) mass, as well as the HPQCD result [2] and the experimental value [1] for comparison.

need to interpolate in $m_{\pi}$ to obtain $L^{*} f_{K}^{\text {ref }}=0.578(9)(12)(6)$ and $L^{*} f_{D}^{\text {ref }}=0.797(9)(16)(9)$. The errors quoted are from statistics, $L^{*}$ scale setting and interpolation, respectively. We find that lattice spacing effects are about $7(5) \%$ in $f_{K}^{\text {ref }}$, but small in $f_{D}^{\text {ref }}$.

\section{$5.3 f_{D_{s}}$ towards the physical point}

To approach the physical point, we take our lightest pion mass, which is $m_{\pi}=234(10)(3)$ $\mathrm{MeV}$ on the E6 ensemble.

As for the quark mass, we interpolate linearly to $m_{D_{s}}=1968 \mathrm{MeV}$ at fixed $\left(m_{K}^{2}-\frac{1}{2} m_{\pi}^{2}\right)$. A plot of $f_{D_{s}}$ as a function of $\left(m_{K}^{2}-\frac{1}{2} m_{\pi}^{2}\right)$ is shown in fig. 1. Extrapolating to the physical point $\left(m_{K}^{2}-\frac{1}{2} m_{\pi}^{2}\right)=0.236(\mathrm{GeV})^{2}$, we obtain our preliminary estimate of $f_{D_{s}}=257(3)(3)(5)(?)$, where the question mark denotes unknown systematic errors, including those coming from the overall scale ambiguity and the quenching of the strange and charm quarks.

We summarize our preliminary findings for $f_{D_{s}}$ in fig. 2, which illustrates that cutoff effects are small (at least for heavy pions), and that the light-quark mass dependence is also small (at least on the coarser lattice). The chiral and continuum extrapolations therefore seem to be well possible.

\section{Summary}

The CLS effort is now simulating very large and fine $N_{f}=2$ lattices, and lattice spacings as small as $a=0.04 \mathrm{fm}$ have become accessible, making fully relativistic charm quarks feasible. As simulations are progressing, lighter sea quarks are also being simulated. 
Our preliminary study of the $D_{s}$ system indicates that cutoff effects are small and under control, but a more precise scale determination is a priority in order to eliminate an important source of systematic error.

With better statistics and more sea and valence quark masses to come, we expect to be able to perform an accurate determination of $f_{D_{s}}$ in the near future.

Acknowledgements. The authors thank Giulia de Divitiis, Björn Leder and Roberto Petronzio for their collaboration in this research project.

We thank NIC/Forschungszentrum Jülich for computer time on the BlueGene/P and BlueGene/L.

This work was supported by the Deutsche Forschungsgemeinschaft in the SFB/TR 09 and under grant HE 4517/2-1, and by the European community through EU contract No. MRTN-CT2006-035482 "FLAVIAnet".

\section{References}

[1] S. Stone, The purely leptonic decays $D^{+} \rightarrow \mu^{+} v$ and $D_{s}^{+} \rightarrow \ell^{+} v$ at CLEO, arXiv:0806.3921.

[2] E. Follana, C. T. H. Davies, G. P. Lepage, J. Shigemitsu, High Precision determination of the $\pi, K, D$ and $D_{s}$ decay constants from lattice QCD, Phys.Rev.Lett. 100 (2008) 062002 [arXiv:0706.1726].

[3] B. A. Dobrescu and A. S. Kronfeld, Accumulating evidence for nonstandard leptonic decays of $D_{s}$ mesons, Phys.Rev.Lett. 100 (2008) 241802 arXiv:0803.0512].

[4] M. Lüscher, Schwarz-preconditioned HMC algorithm for two-flavour lattice QCD, Comput.Phys.Commun. 165 (2005) 199 hep-lat/ 0409106 ; M. Lüscher, Local coherence and deflation of the low quark modes in lattice QCD, JHEP 0707 (2007) 081 [arXiv:0 06.2298 ].

[5] L. Del Debbio, L. Giusti, M. Lüscher, R. Petronzio and N. Tantalo, QCD with light Wilson quarks on fine lattices. I: First experiences and physics results, JHEP 0702 (2007) 056 [hep-lat/ 061056.

[6] M. Della Morte et al. (Alpha collaboration), Preparing for $N_{f}=2$ simulations at small lattice spacings, PoS(LAT2007) 255 [arXiv:0710.1263].

[7] L. Del Debbio, L. Giusti, M. Lüscher, R. Petronzio and N. Tantalo, $Q C D$ with light Wilson quarks on fine lattices. II: DD-HMC simulations and data analysis, JHEP 0702 (2007) 082 hep-lat/0701009].

[8] M. Della Morte, R. Hoffmann and R. Sommer, Non-perturbative improvement of the axial current for dynamical Wilson fermions, JHEP 0503 (2005) 029 hep-lat / 0503003 .

[9] M. Della Morte, R. Sommer and S. Takeda, On cutoff effects in lattice QCD from short to long distances, arXiv:0807.1120.

[10] M. Della Morte et al. (Alpha collaboration), Non-perturbative quark mass renormalization in two-flavor QCD, Nucl.Phys. B 729 (2005) 117 [hep-lat/0507035].

[11] J. Heitger et al., in preparation.

[12] J. Rolf and S. Sint (Alpha collaboration), A precise determination of the charm quark's mass in quenched QCD, JHEP 0212 (2002) 007 [hep-ph / 0209255$].$ 